\title{
Surgical Capacity Surveys: A Call for Papers on the Next Steps for Moving Beyond Descriptive Data
}

\author{
Charles Mock
}

Published online: 7 February 2015

(C) Société Internationale de Chirurgie 2015

Growing attention is being focused on global surgical issues, with an increasing evidence base being generated in both the surgical and global health literatures. A component of this research includes assessments of the capacity to deliver surgical care in individual hospitals and across networks of hospitals in many low- and middle-income countries. These articles have addressed human resources (skills, training, staffing), physical resources (equipment, supplies), and infrastructure. The article by Lucas et al. summarizes this literature to date [1]. After an extensive search, the authors document a total of 28 studies from 17 countries that use a variety of inter-related tools to evaluate such surgical capacity. The authors restrict their search to articles on surgical capacity in general and allude to, but do not summarize, data from studies on pediatric surgery, trauma care, or other sub-components of overall surgical capacity.

The literature on surgical capacity has been robust and has addressed a wide spectrum of economic levels and geography (with the exception of North Africa and the Middle-East). These articles have helped to bring attention to the frequent low level of development of surgical capacity in many countries. They have demonstrated interesting differences between countries, even countries at the same economic levels. They have identified specific deficits to work on.

However, only a small number of the articles (seven articles representing four countries) report on actual use of the surveys in making improvements. These include

Charles Mock-Associate Editor, World Journal of Surgery

C. Mock $(\bowtie)$

Seattle, WA, USA

e-mail: cmock@uw.edu articles which document that the results of earlier surveys were used in the formulation of interventions to strengthen surgical services, such as improved training programs or development of a national initiative for improving surgical care. However, only two articles used the survey tools to monitor changes in capacity over time, whether in response to interventions or just as the result of secular trends. No articles report on changes in either process of care or its health outcomes (morbidity or mortality).

The World Journal of Surgery feels that it is time to take the next step on surgical capacity surveys and calls for papers that do so. We would be especially interested in considering articles that

- Use capacity assessments to monitor changes over time.

- Report on specific interventions, using the capacity surveys to analyze the results of these interventions. Rigorous study designs with control groups would be of special interest.

- Report on changes in process of care and health outcomes (morbidity or mortality) in response to either interventions to improve surgical capacity or at least in response to corresponding changes in that capacity documented by the surveys.

Articles that report descriptive data at one point in time will be of less interest as there is already a robust literature on that topic. However, descriptive articles that report on one or more of the following additional details would add to the existing literature and hence would be considered:

- In-depth assessment of underlying causes of deficiencies or contributing factors, such as barriers created by user fees or specific technological problems (e.g., specific mechanical or engineering issues on frequently problematic pieces of equipment). These articles should 
have specific policy implications that are well supported by the data presented.

- Articles from North Africa or the Middle-East, which have not yet been represented in the surgical capacity literature.

- Other novel aspects of capacity surveys that have not been present in and that contribute to the existing literature.

Any articles submitted on these topics should adhere to the World Journal of Surgery policy that data from lowand middle-income countries should have at least one (preferably more) co-author from that country (preferably as lead author). If it is not possible to have at least one coauthor from that country, the reason for this should be explained in the cover letter.

\section{Reference}

1. Carlson L, Lin J, Ameh E et al (2015) Moving from data collection to application: a systematic literature review of surgical capacity assessments and their applications. World J Surg. doi:10.1007/ s00268-014-2938-8 